2011

\title{
A Non-Radial Oscillation Model For Pulsar State Switching
}

R. Rosen

M.A. McLaughlin

S.E. Thompson

Follow this and additional works at: https://researchrepository.wvu.edu/faculty_publications

\section{Digital Commons Citation}

Rosen, R.; McLaughlin, M. A.; and Thompson, S. E., "A Non-Radial Oscillation Model For Pulsar State Switching" (2011). Faculty Scholarship. 4.

https://researchrepository.wvu.edu/faculty_publications/4 


\title{
A NON-RADIAL OSCILLATION MODEL FOR PULSAR STATE SWITCHING
}

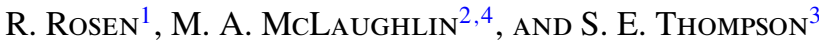 \\ ${ }^{1}$ NRAO, P.O. Box 2, Green Bank, WV 24944, USA; rrosen@nrao.edu \\ 2 Department of Physics, West Virginia University, 436 Hodges Hall, Morgantown, WV 26506, USA \\ ${ }^{3}$ SETI Institute/NASA Ames Research Center, M/S 244-30, Moffett Field, CA 94035, USA \\ Received 2010 October 11; accepted 2010 December 30; published 2011 January 21
}

\begin{abstract}
Pulsars are unique astrophysical laboratories because of their clock-like timing precision, providing new ways to test general relativity and detect gravitational waves. One impediment to high-precision pulsar timing experiments is timing noise. Recently, Lyne et al. showed that the timing noise in a number of pulsars is due to quasi-periodic fluctuations in the pulsars' spin-down rates and that some of the pulsars have associated changes in pulse profile shapes. Here we show that a non-radial oscillation model based on asteroseismological theory can explain these quasi-periodic fluctuations. Application of this model to neutron stars will increase our knowledge of neutron star emission and neutron star interiors and may improve pulsar timing precision.
\end{abstract}

Key words: pulsars: general - stars: neutron - stars: oscillations (including pulsations) - white dwarfs

\section{INTRODUCTION}

Pulsars have long been known as superb laboratories for fundamental physics due to their timing precision and stable pulse profiles. Timing observations of pulsars have resulted in the best tests of general relativity in the strong-field regime, the most precise stellar mass measurements, and the most constraining limits on gravitational wave sources in the nanoHertz regime through "pulsar timing array" experiments (e.g., Jenet et al. 2006; Kramer et al. 2006). One significant limitation for all of these studies is timing noise, or non-random variations in the residuals between measured and model-predicted arrival times. Lyne et al. (2010) recently showed that the timing noise in 17 pulsars is due to the pulsar's spin-down rate fluctuating between different values in a quasi-periodic manner. Furthermore, for six of these pulsars, the changes in the spin-down rate directly correlate to changes in the pulse shape. While the pulsars described in Lyne et al. (2010) have long periods, some millisecond pulsars, such as PSR B1931+27, also exhibit timing noise and may show similar but less obvious effects. This behavior offers a possible prescription for removing timing noise from data and dramatically increasing timing stability, facilitating projects such as the direct detection of gravitational waves through pulsar timing. In this Letter, we present a model for this behavior based on nonradial oscillations. We outline the model in Section 2, discuss underlying assumptions in Section 3, describe the implications for the pulsars discussed in Lyne et al. (2010) in Section 4, and present conclusions in Section 5.

\section{A NON-RADIAL OSCILLATION MODEL}

We show that a non-radial oscillation model can explain all the long-period variations described in Lyne et al. (2010). In our model, based on asteroseismological theory (Dziembowski 1977), non-radial oscillations of high spherical degree $(\ell)$ are aligned to the magnetic axis of the neutron star (Clemens \& Rosen 2004). Between any two adjacent nodal lines, displacements of material move horizontally along the stellar surface toward and away from the magnetic pole, as shown in the schematic of Figure 1. Jessner et al. (2001) showed that thermal

\footnotetext{
4 Also adjunct at the National Radio Astronomy Observatory.
}

emission of electrons dominates the rate at which charged particles flow from the surface into the magnetosphere. Non-radial oscillations, and their associated velocities, will vary the amount of thermal emission by creating regions of local heating due to compression between the nodal lines, thus increasing the flow of charged particles into the magnetosphere and modulating the radio emission (Strohmayer 1992).

The situation is analogous to behavior seen in white dwarf stars. Some white dwarfs are $g$-mode, multi-periodic pulsators that tend to excite a select number of pulsation modes, as shown in Figure 2. In adiabatic oscillations, local heating of the stellar surface coincides with the pulsation maximum during the compression phase of the pulsation. A quarter cycle later, material will be moving away at a maximum velocity. The displacements responsible for surface heating and flux changes in oscillating white dwarfs are primarily non-radial because of their high surface gravity (Robinson et al. 1982) and have measured radial velocity variations that can be interpreted as the horizontal velocities (van Kerkwijk et al. 2000; Thompson et al. 2003). In neutron stars, we expect that the amplitude of the displacements follows surface thermal variations caused by non-radial oscillations of the neutron star. As with the white dwarf stars, an increase in the velocities can result in increased thermal emission from the star.

In our non-radial oscillation model, each frequency of oscillation has its own periodic velocity (or displacement) amplitude. In the pulsars we have studied to date, the amplitude of the displacements dictates the average pulse shape (Rosen \& Clemens 2008; Rosen \& Demorest 2011), but as the amplitude of the velocities grows, the average pulse shape can change (Clemens \& Rosen 2008). In these pulsars, through fitting a non-radial oscillation model to their drifting subpulses, we have also found $\ell$ to be large compared to that seen in white dwarf stars, consistent with their smaller size. For pulsars B0809+74 and B0943+10, $\ell$ values range from $126 \leqslant \ell \leqslant 133$ (Rosen \& Demorest 2011) and $385 \leqslant \ell \leqslant 875$ (Rosen \& Clemens 2008), respectively. In white dwarf stars, modes with high $\ell$ fade from view because of geometric cancellation of the stellar surface, leaving only modes of low $\ell$ ( $\ell \leqslant 4$; Yeates et al. 2005; Thompson et al. 2004, 2008).

By fitting a non-radial oscillation model to the drifting subpulses in PSRs B0943+10 and B0809+74, we measured the 

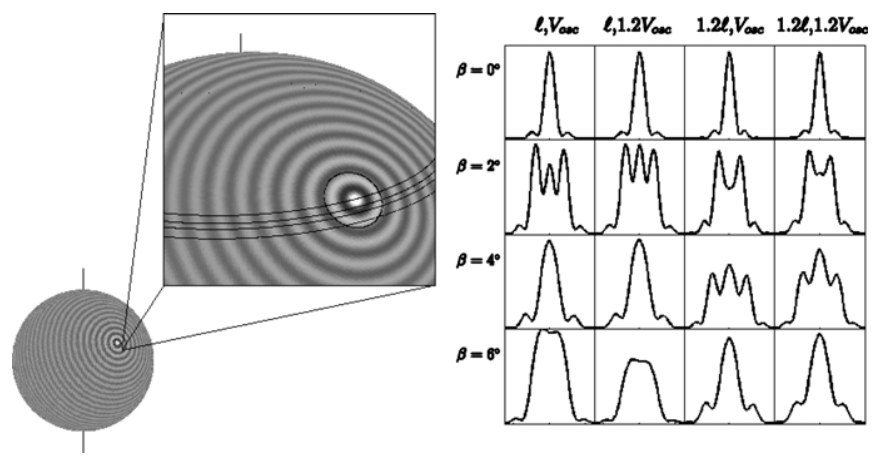

Figure 1. Schematic: a map of the non-radial oscillations on a pulsar. The circle around the magnetic pole in the enlarged view denotes the boundary of the emitting region. Dark regions indicate negative displacements and light regions indicate positive ones. After a half cycle of the oscillations, the dark regions would be light and vice versa, but the nodal lines (as defined by $\ell$ ) separating them would remain unchanged, except for rotation of the whole pattern about the rotation axis of the star. The magnetic cap is crossed by four representative sight lines, where $\beta$ is the angle between our sight line and the magnetic cap. The listed values for $\beta$ are equally spaced. Panels: for each sight line, the columns show the corresponding average profile (i.e., intensity vs. pulse phase) for various values of $V_{\mathrm{osc}}$ and $\ell$.

pulsation period to be on the order of $30-50 \mathrm{~ms}$ (Rosen \& Clemens 2008; Rosen \& Demorest 2011). These values for the pulsation period are consistent with core $g$-modes (Reisenegger \& Goldreich 1992). However, core $g$-modes require large excitation energies, and therefore surface $g$-modes are a possibility (Strohmayer 1993) as they have lower energies and larger surface amplitudes than the core modes (McDermott et al. 1988). The period predictions for core $g$-modes in neutron stars range from a minimum value of $10 \mathrm{~ms}$ (Reisenegger \& Goldreich 1992) to 2-88 s (McDermott et al. 1988) depending on the model for the structure and composition of the stellar interior. Surface $g$-modes have periods in the $40-400 \mathrm{~ms}$ range (McDermott et al. 1988). While the pulsation periods calculated for most models are calculated assuming low $\ell$, the period of the mode decreases for higher spherical degree (McDermott et al. 1988). It is unlikely that the pulsation modes we see in PSRs B0943+10 and B0809+74 are $p$-modes as these modes have periods on the order of tenths of milliseconds (McDermott et al. 1988) and have overtones with shorter periods, whereas the overtones of $g$-modes have longer periods.

The two oscillation driving mechanisms for white dwarf stars are the $\kappa-\gamma$ mechanism and convective driving. For pulsating DOV stars like PG1159-035, characterized by an atmosphere that lacks hydrogen but shows helium, carbon, and oxygen absorption lines, the $\kappa-\gamma$ mechanism drives the pulsations as the opacity varies steeply with pressure (Starrfield et al. 1983; Córsico et al. 2006; Cox 2003). In DAV and DBV stars, which have atmospheres dominated by hydrogen or helium, respectively, convective motions drive pulsations (Brickhill 1991; Goldreich \& Wu 1999; Wu \& Goldreich 1999).

Driving mechanisms for neutron star oscillations are different and less well understood. Jessner et al. (2001) show that thermal and field emission from the neutron star surface can accelerate electrons along open field lines with the formation of a vacuum gap. Therefore, oscillations should be related to thermal variations at the neutron star surface, as would be produced by $g$-modes. The high spherical degree that we observe in the data (and is predicted by our model) suggests that the pulsation driving energy is concentrated in a small area on the surface of the star. Otherwise it would average away over multiple surface zones (Clemens \& Rosen 2004). In one possible scenario, the

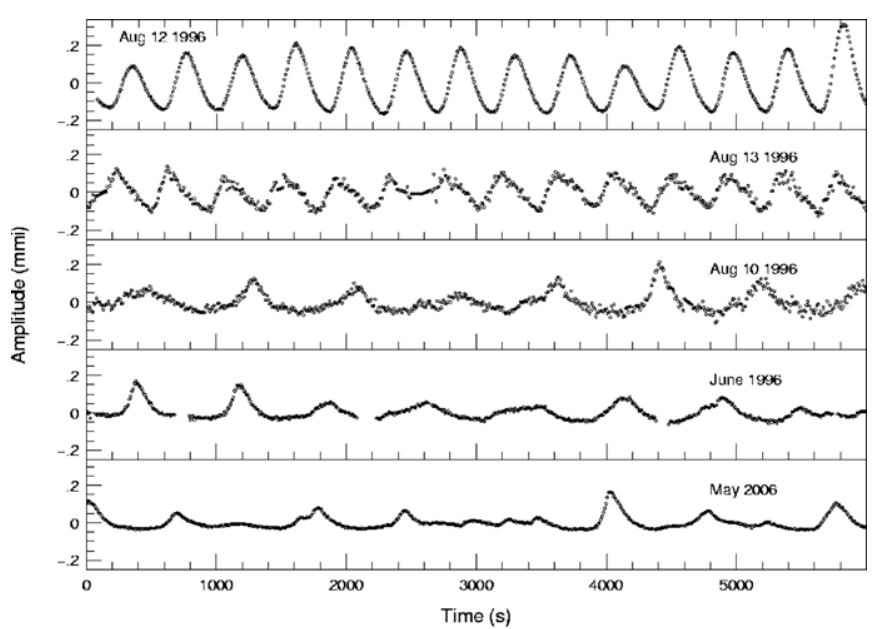

Figure 2. Flux variations of GD358, a helium atmosphere pulsating white dwarf star, observed on different nights (Provencal et al. 2009, figure reproduced with the authors' permission). For two nights in 1996, the light curve of GD358 drastically changed. Unlike during all other observations of this star, all the pulsation energy was pumped into the shortest period modes.

source of the driving energy is at the magnetic cap and the particle emission exerts a torque on the open field lines, which is coupled to the rest of the star via magnetic and mechanical dissipation. If the magnetic field is coupled (even weakly) to the surface and displaces material laterally, the torque on the open field lines results in a displacement of material on the polar cap. The heating that results can increase particle emission, which then increases the torque, and drives the oscillations (Clemens \& Rosen 2004).

Other possibilities for driving mechanisms involve direct shaking of field lines (Boriakoff 1976), thermal stratification (McDermott et al. 1983), chemical stratification (Finn 1987), and stratification in the core (Reisenegger \& Goldreich 1992). The gravitational radiation damping timescale for $g$-modes exceeds the age of the universe and is therefore a negligible effect (McDermott et al. 1983; Reisenegger \& Goldreich 1992). The pulsations we observe in PSRs B0943+10 and B0809+74 appear to persist on hour to month (if not longer) timescales and any driving mechanism must be in agreement with these observations. Other neutron star oscillations have been detected in the form of quasi-periodic oscillations (QPOs) from soft gamma-ray repeaters. These have been interpreted as torsional shear modes; the frequencies of QPOs are too high to originate from $g$-modes (Watts \& Strohmayer 2007).

\section{STANDARD MODEL ASSUMPTIONS}

In the standard pulsar model (Goldreich \& Julian 1969), the magnetosphere is considered to be "force-free" in that the electromagnetic energy density dominates all other dissipative forces and that radiative emission has a negligible effect on the spin-down rate (Arons 2009). Instead, the configuration of the magnetosphere, based on the polar cap cascade zone and the current density within the open field lines, and its change over time dictate the pulsar spin-down rate (Timokhin 2006, 2010; Arons 2009). Recently, Timokhin (2010) speculates that the magnetospheres of some pulsars can have several quasi-stable states with different configurations and that the switching of the magnetosphere between these states can result in the observed mode changes and nulls. Timokhin (2010) further notes that each state would have a different spin-down rate. 
Table 1

List of Pulsars Which Show Changes in the Spin-down Rate with Possible Classifications with Respect to Non-radial Oscillation Properties

\begin{tabular}{lccc}
\hline \hline Scenario 1 & Scenario 1 & Scenario 2 & Scenario 3 \\
\hline$\Delta \dot{P}<\Delta \dot{P}_{\text {crit }}$ & $\Delta \dot{P}>\Delta \dot{P}_{\text {crit }}$ & $\Delta \dot{P}<\Delta \dot{P}_{\text {crit }}$ & $\Delta \dot{P}>\Delta \dot{P}_{\text {crit }}$ \\
$\begin{array}{l}\text { No Pulse Shape Changes } \\
\text { Small } \Delta V_{\text {osc }}, \text { No } \Delta \ell\end{array}$ & $\begin{array}{c}\text { Small Pulse Shape Changes } \\
\text { Large } \Delta V_{\text {osc }}, \text { No } \Delta \ell\end{array}$ & $\begin{array}{c}\text { Pulse Shape Changes } \\
\text { No } \Delta V_{\text {osc }}, \Delta \ell\end{array}$ & $\begin{array}{c}\text { Large Pulse Shape Changes } \\
\Delta V_{\text {osc }}, \Delta \ell\end{array}$ \\
\hline B1642-03 & B2035+36 & J2043+2740 & B1931+24 \\
B1903+07 & $\ldots$ & B1822-09 & $\ldots$ \\
B1839+09 & $\ldots$ & B1540-06 & $\ldots$ \\
B2148+63 & $\ldots$ & B1828-11 & $\ldots$ \\
B1818-04 & $\ldots$ & B0740-28 & $\ldots$ \\
B0950+08 & $\ldots$ & $\ldots$ & $\ldots$ \\
B1714-34 & $\ldots$ & $\ldots$ & $\ldots$ \\
B1907+00 & $\ldots$ & $\ldots$ & $\ldots$ \\
B1826-17 & $\ldots$ & $\ldots$ & $\ldots$ \\
B0919+06 & $\ldots$ & $\ldots$ & $\ldots$ \\
B1929+20 & $\ldots$ & $\ldots$ & $\ldots$ \\
\hline
\end{tabular}

Timokhin (2007) shows that oscillation modes of high spherical degree and non-vanishing velocities at the surface can alter the local Goldreich-Julian (GJ) charge density (Goldreich \& Julian 1969). The GJ charge density, while not a contributing factor to the spin-down rate in the force-free model, is then a combination of the charge density due to rotation and the charge density due to oscillations. In this Letter, we assume that, like white dwarf stars, neutron star oscillations can change with both amplitude and spherical degree, therefore affecting the total charge density and the magnetospheric configuration. If the charge density is at least partially responsible for the energy loss of the pulsar and/or the change in pulsation mode changes the magnetospheric configuration, we expect to observe a change in the spin-down rate.

\section{THEORETICAL PREDICTIONS}

Under some models we expect for the spin-down rate and the pulse shape to change with both spherical degree and the amplitude of the velocities of the oscillations, $V_{\text {osc }}$. Timokhin (2007) calculates the GJ charge density for neutron star oscillations assuming the standard pulsar model of a rotating magnetized conducting sphere surrounded by plasma, and explores the excitation of oscillations by neutron star glitches. He shows that oscillation modes of high spherical degree and non-vanishing velocities at the surface can alter the local GJ charge density $\left(\rho_{\mathrm{GJ}}\right)$, where $\rho_{\mathrm{GJ}} \sim \ell\left(V_{\mathrm{osc}} / c\right)\left(B / 4 \pi R_{\mathrm{NS}}\right)$. The charge density will affect the accelerating electric field. For the oscillations to be strong enough to influence the particle distribution, resulting in effects that we can observe, Timokhin (2007) suggests the spherical degree is approximately several hundreds, which is in agreement with our independent measurements (Rosen \& Clemens 2008; Rosen \& Demorest 2011). Because more particles leave the stellar surface when the charge density is greater, we expect the spin-down rate to increase with either (1) increasing $\ell$, (2) increasing $V_{\text {osc }}$, or (3) increasing $\ell$ and $V_{\text {osc }}$. Assuming that the current density in the magnetosphere is given by Equation (1) (Lorimer \& Kramer 2004, p. 64), the charge density is directly related to the spin-down rate by

$$
\frac{\rho_{\mathrm{GJ}}}{e}=-\frac{\Omega B}{2 \pi c e} \simeq 7 \times 10^{10} \mathrm{~cm}^{-3}\left(\frac{P}{s}\right)^{-1 / 2}\left(\frac{\dot{P}}{10^{-15}}\right)^{1 / 2},
$$

where $\Omega$ is the angular rotation frequency, $B$ is the magnetic field, $P$ is the spin period of the pulsar, and $\dot{P}$ is the spin-down rate. Note that this assumption is not in agreement with the simple force-free model. We calculate the fractional change in charge density using the change in the spin-down rate and then use this to calculate $\rho_{2} / \rho_{1}=\ell_{2} V_{\text {osc }, 2} / \ell_{1} V_{\text {osc, } 1}$. For the pulsar with the largest change in the spin-down rate, PSR B1931+24, the value of $\ell V_{\text {osc }}$ will change by $20 \%$; for PSR B1929+10, which has the smallest change in the spin-down rate, $\ell V_{\text {osc }}$ will change by $0.15 \%$. To make these estimates, we assume that the contribution to the charge density due to oscillations is much greater than the contribution due to rotation. Figure 1 illustrates how changes in these parameters affect the pulse shapes and Table 1 shows our best guesses for the classifications of the pulsars in Lyne et al. (2010) into the different scenarios.

In the first scenario where $V_{\text {osc }}$ changes but $\ell$ remains constant, pulsars fall into two categories: small and large velocity oscillations. For small velocity oscillations, the change in the spin-down rate is less than some value, $\Delta \dot{P}_{\text {crit }}$, and is accompanied by little or no change in the pulse shape. With the small sample of pulsars that show this behavior in Lyne et al. (2010), we cannot determine the precise demarcation point between large and small changes in the spin-down rate, but estimate it to be $\Delta \dot{P}_{\text {crit }} \sim 10 \%$ by inspection of those pulsars which show pulsar shape changes. The pulsars in the first column of Table 1 fall into this category. Many stable pulsating white dwarf stars have subtle changes in amplitude over time, thereby changing $V_{\text {osc }}$ but not $\ell$ (Provencal et al. 2009; Vuille 2000; Handler et al. 2008). When the amplitude of a single mode changes, both its flux and velocity amplitudes change. If amplitude changes occur on a neutron star, the change in $V_{\text {osc }}$ will also change the spin-down rate. A periodic change in amplitude will result in a periodic change in the spin-down rate. Because the spherical harmonic is responsible for the average pulse shape and $\ell$ is constant, the average pulse shows little or no change with varying spin-down rate.

Pulsars with large velocity oscillations have changes in the spin-down rates greater than $\Delta \dot{P} \dot{P}_{\text {crit }}$ with changes in the pulse shape, such as B2035+36, are listed in the second column of Figure 1. In the small sample of pulsars we have quantitatively fit, the average pulse shape is dominated by the amplitude of the displacements (Clemens \& Rosen 2008). However, when the relative amplitudes of the velocities and displacements become dominated by the velocities, the pulse shape can change and we may observe a corresponding change in the polarization behavior as well (Clemens \& Rosen 2008). PSR B2035+36 shows a sudden large change in the spin-down rate. Analogously, the white dwarf star GD358 suddenly pumped all of its energy 
into the shortest period modes, increasing the overall amplitude and changing the pulsation characteristics on a timescale of hours as shown in Figure 2 (Kepler et al. 2003; Provencal et al. 2009). Such exchanges in the energy between pulsations on white dwarfs are not always so dramatic but are common (Kleinman et al. 1998; Vuille 2000; Wu \& Goldreich 2001).

Pulsars that have changes in the spin-down rate less than $\Delta \dot{P}_{\text {crit }}$ accompanied by changes in the pulse shape, like those listed in Column 3 of Table 1, fall into the second scenario, where $\ell$ varies but $V_{\text {osc }}$ remains constant, as illustrated in the third column of Figure 1. Periodic changes in $\ell$ will modulate both the spin-down rate, the pulsation period, and the average pulse profile. White dwarf stars are known to variably excite pulsations of $\ell$, varying from $1 \leqslant \ell \leqslant 4$ (Kepler et al. 2003; Thompson et al. 2008; Clemens et al. 2000). When oscillations of different $\ell$ are excited on a pulsar, the relationship between the nodal structure to the pulse width is more complicated than a simple proportion due to the geometry of the star and our viewing angle relative to the magnetic pole. While it is easy to assume that as the area between nodal lines gets larger the pulse width increases; for certain geometries, the pulse width can actually decrease, as in PSR J2043+2740 and as illustrated in Figure 1. Radio polarization data would allow us to determine the geometry and the amount by which $\ell$ is changing.

The third scenario, where both $V_{\text {osc }}$ and $\ell$ change, can also be possible through the transfer of energy between modes. This is shown in the fourth column of Figure 1 and Table 1 . If both $V_{\text {osc }}$ and $\ell$ vary, we expect larger changes in the spin-down rate and pulse width, like that seen in PSR B1931+24. If $V_{\text {osc }}$ and $\ell$ are changing at different rates, we also expect to see more complicated behavior including beat frequencies, which would result in complicated patterns in the spin-down rate and pulse shape. Extrapolating the more complex behavior of unstable white dwarf pulsators to neutron stars, we would expect to see multiple, possibly simultaneous pulsation modes. The changes in the light curves in white dwarf stars are not necessary gradual or sinusoidal and therefore we do not necessarily expect gradual changes in the spin-down and pulse shape in neutron stars. The velocity and spherical degree could change depending on which mode is dominating the pulsation spectrum.

All the scenarios above can explain how the spin-down rate can change periodically with or without accompanying changes in the pulse profile. It may be that some pulsars do not exhibit any changes in the spin-down rate with time but still show pulsations. These objects would have stable pulsation modes. Stable white dwarf pulsators can have several pulsation modes present simultaneously and tend to be dominated by a few pulsations (Yeates et al. 2005; Mullally et al. 2008). We speculate that neutron stars which do not exhibit changes in the spin-down rate or period show no changes in $V_{\text {osc }}$ or $\ell$, but may have several pulsations modes present. PSRs B0943+10 and B0809+74 have both been quantitatively fit to an oscillation model but do not exhibit any known changes in the spin-down rate (Rosen \& Clemens 2008; Rosen \& Demorest 2011). Just as only a percentage of white dwarf stars exhibit pulsations, we expect only a fraction of neutron stars to show oscillations.

\section{CONCLUSION}

In summary, we have shown that the fluctuations in the spindown rate described in Lyne et al. (2010) can be explained by a non-radial oscillation model. Our model is not complete, as little is understood about pulsar magnetospheres and there is no clear mechanism for driving the oscillations. However, our oscillation model does not require invoking an external influence to explain the periodicity, as in Cordes \& Shannon (2008), and is based on observations of other well-studied stars. This will lead to better understanding of pulsar emission physics and may enable optimal removal of effects due to non-radial oscillations in pulsar timing data, increasing the usefulness of pulsars as fundamental physics laboratories.

M.A.M. is supported by WVEPSCOR, the Research Corporation, the Sloan Foundation, and the National Science Foundation. The National Radio Astronomy Observatory is a facility of the National Science Foundation operated under cooperative agreement by Associated Universities, Inc. We also thank Duncan Lorimer and Xavier Seimens for helpful comments on the manuscript.

\section{REFERENCES}

Arons, J. 2009, in Neutron Stars and Pulsars, ed. W. Becker (ASSL Vol. 357; Berlin: Springer), 373

Boriakoff, V. 1976, ApJ, 208, L43

Brickhill, A. J. 1991, MNRAS, 251, 673

Clemens, J. C., \& Rosen, R. 2004, ApJ, 609, 340

Clemens, J. C., \& Rosen, R. 2008, ApJ, 680, 664

Clemens, J. C., van Kerkwijk, M. H., \& Wu, Y. 2000, MNRAS, 314, 220

Cordes, J. M., \& Shannon, R. M. 2008, ApJ, 682, 1152

Córsico, A. H., Althaus, L. G., \& Miller Bertolami, M. M. 2006, A\&A, 458, 259

Cox, A. N. 2003, ApJ, 585, 975

Dziembowski, W. 1977, Acta Astron., 27, 203

Finn, L. S. 1987, MNRAS, 227, 265

Goldreich, P., \& Julian, W. H. 1969, ApJ, 157, 869

Goldreich, P., \& Wu, Y. 1999, ApJ, 511, 904

Handler, G., Romero-Colmenero, E., Provencal, J. L., Sanchawala, K., Wood, M. A., Silver, I., \& Chen, W.-P. 2008, MNRAS, 388, 1444

Jenet, F. A., et al. 2006, ApJ, 653, 1571

Jessner, A., Lesch, H., \& Kunzl, T. 2001, ApJ, 547, 959

Kepler, S. O., et al. 2003, A\&A, 401, 639

Kleinman, S. J., et al. 1998, ApJ, 495, 424

Kramer, M., et al. 2006, Science, 314, 97

Lorimer, D. R., \& Kramer, M. (ed.) 2004, Handbook of Pulsar Astronomy, Cambridge Observing Handbooks for Research Astronomers, Vol. 4 (Cambridge: Cambridge Univ. Press)

Lyne, A., Hobbs, G., Kramer, M., Stairs, I., \& Stappers, B. 2010, Science, 329, 408

McDermott, P. N., van Horn, H. M., \& Hansen, C. J. 1988, ApJ, 325, 725

McDermott, P. N., van Horn, H. M., \& Scholl, J. F. 1983, ApJ, 268, 837

Mullally, F., Winget, D. E., De Gennaro, S., Jeffery, E., Thompson, S. E., Chandler, D., \& Kepler, S. O. 2008, ApJ, 676, 573

Provencal, J. L., et al. 2009, ApJ, 693, 564

Reisenegger, A., \& Goldreich, P. 1992, ApJ, 395, 240

Robinson, E. L., Kepler, S. O., \& Nather, R. E. 1982, ApJ, 259, 219

Rosen, R., \& Clemens, J. C. 2008, ApJ, 680, 671

Rosen, R., \& Demorest, P. 2011, ApJ, in press (arXiv:1012.6020)

Starrfield, S. G., Cox, A. N., Hodson, S. W., \& Pesnell, W. D. 1983, ApJ, 268, L27

Strohmayer, T. E. 1992, ApJ, 388, 138

Strohmayer, T. E. 1993, ApJ, 417, 273

Thompson, S. E., Clemens, J. C., van Kerkwijk, M. H., \& Koester, D. 2003, ApJ, 589,921

Thompson, S. E., Clemens, J. C., van Kerkwijk, M. H., O’Brien, M. S., \& Koester, D. 2004, ApJ, 610, 1001

Thompson, S. E., van Kerkwijk, M. H., \& Clemens, J. C. 2008, MNRAS, 389, 93

Timokhin, A. N. 2006, MNRAS, 368, 1055

Timokhin, A. N. 2007, Ap\&SS, 308, 345

Timokhin, A. N. 2010, MNRAS, 408, L41

van Kerkwijk, M. H., Clemens, J. C., \& Wu, Y. 2000, MNRAS, 314, 209

Vuille, F. 2000, MNRAS, 313, 170

Watts, A. L., \& Strohmayer, T. E. 2007, Adv. Space Res., 40, 1446

Wu, Y., \& Goldreich, P. 1999, ApJ, 519, 783

Wu, Y., \& Goldreich, P. 2001, ApJ, 546, 469

Yeates, C. M., Clemens, J. C., Thompson, S. E., \& Mullally, F. 2005, ApJ, 635, 1239 